Horizons philosophiques

\title{
Le droit du travail comme révélateur du conflit des normes juridiques et des valeurs
}

\section{Christine Noël}

Volume 12, numéro 2, printemps 2002

Valeurs et modernité

URI : https://id.erudit.org/iderudit/801209ar

DOI : https://doi.org/10.7202/801209ar

Aller au sommaire du numéro

Éditeur(s)

Collège Édouard-Montpetit

ISSN

1181-9227 (imprimé)

1920-2954 (numérique)

Découvrir la revue

Citer cet article

Noël, C. (2002). Le droit du travail comme révélateur du conflit des normes juridiques et des valeurs. Horizons philosophiques, 12(2), 75-85.

https://doi.org/10.7202/801209ar d'utilisation que vous pouvez consulter en ligne.

https://apropos.erudit.org/fr/usagers/politique-dutilisation/ 


\section{LE DROIT DU TRAVAIL COMME RÉVÉLATEUR DU CONFLIT DES NORMES JURIDIQUES ET DES VALEURS}

Prendre le droit comme objet d'interrogation philosophique relève à bien des égards de la gageure, à plus forte raison quand il s'agit du droit du travail. Gageure épistémologique, tout d'abord! Sous l'impact successif du jusnaturalisme puis du positivisme juridique, le droit a été assimilé à un système formel de normes arbitraires. Le droit, réduit au seul droit positif, n'est que l'ensemble des législations en vigueur à une époque et dans des lieux déterminés. Le positivisme juridique a ainsi eu comme conséquence directe de rendre non pertinente la question du juste. Un système juridique ne saurait être qualifié de juste ou d'injuste qu'en référence à des valeurs ou à des normes qui lui préexisteraient et qui découleraient d'un droit naturel universel et éternel, qui s'imposerait au législateur. Or à partir du moment où la législation est appréhendée non comme quelque chose de subordonné au droit naturel, mais comme un ensemble de décisions arbitraires, destinées à réglementer des faits et à les orienter, le droit positif ne peut plus être évalué, jugé qu'en fonction de lui-même, en tant que système formel, et en fonction de la société qu'il est chargé de réglementer. La question du caractère juste ou injuste d'une législation est éclipsée par des questions relatives à la cohérence, à la congruence et à la pertinence d'un système législatif. Ce type de réflexion ne peut être conduit qu'à partir d'une analyse des faits législatifs et des faits empiriques, et il relève davantage du ressort du juriste et du sociologue que de la sphère de compétence du philosophe. II n'y a rien d'étonnant à ce que le philosophe ait été dépossédé par le juriste de toute prétention légitime à questionner le droit.

Il est caractéristique que la philosophie du droit soit enseignée en France par des juristes, dans le cadre des Facultés de droit, et que cette discipline ait cédé sa place dans la formation des jeunes juristes à une «théorie du droit", chargée d'expliciter les notions et les structures d'ensemble du droit positif. L'apport kelsénien a largement favorisé cette opération de délestage épistémologique 1 . Pourtant, il n'explique pas ce phénomène à lui seul.

1. A. Renaut décrit ce processus dans Qu'est-ce que le droit? Aristote, Wolff et Fichte, Paris, Prétextes, Vrin, 1992, introduction, p. 8 et suivantes. 
Dans le même temps, les philosophes ont eux-mêmes contribué à jeter le soupçon sur toute réflexion qui prenait le droit et le juste comme objets. L'historicisme, réduisant le droit à un universel abstrait, qui revêt des visages successifs et évanescents, simples figures de l'épistémé d'une époque, le condamne à n'être qu'un vocable chargé d'unifier d'une manière purement illusoire des expériences juridiques incommensurables. Si comme le rappelle Michel Miaille, le droit, défini comme "un système de règles ${ }^{2}$ de comportement, énoncés et appliqués au sein de la société dans des modalités spécifiques, organisant au nom de certaines valeurs, un ordre et une logique de régulation sociale", est un "produit culturel» en ce qu'il est effectivement construit par la société qui lui donne sa cohérence et sa logique de fonctionnement, nous ne saurions en aucun cas échapper à une compréhension historique du phénomène juridique ${ }^{3}$. II suffit d'ailleurs de prendre acte des différences de législation en fonction des cultures pour souscrire à la nécessité d'une approche historique et analytique du droit. Mais cette approche, qui privilégie l'explication à la compréhension, épuise-t-elle pour autant tous les modes de questionnement qu'il est possible d'appliquer au juridique? La réduction de toute interrogation du droit à une analyse, quand bien même critique des systèmes juridiques existants est porteuse d'un mépris implicite du politique et de sa fonction dans la mesure où elle implique l'abandon du projet de comprendre le juridique pour se contenter de l'expliquer. Comprendre un phénomène, c'est en saisir le sens dans sa globalité par une approche intuitive et synthétique; l'expliquer, c'est au contraire privilégier l'ordre discursif et analytique. Mais expliquer sans comprendre ne relève-t-il pas de l'illusion, voire d'une certaine forme de lâcheté épistémologique?

Dans ce contexte intellectuel, la simple question «qu'est-ce que le droit?" préalable méthodologique à toute investigation philosophique en matière juridique relèverait tant pour les juristes que pour les philosophes d'une "epistémé aussi dépassée que pour l'être l'âge métaphysique dont elle serait solidaire ${ }^{4 »}$.

2. Une règle est une formule qui indique ou qui prescrit une conduite dans une situation ou dans un domaine déterminés. Comme l'instrument qui sert à tracer des traits droits, une règle indique le "droit chemin» pour parvenir à certaines fins. F. Hayek définit les règles comme des "propensions ou des dispositions à agir ou à ne pas agir d'une certaine façon qui se manifeste dans une pratique ou une coutume". in Droit, Législation et Liberté, tome I, Règles et ordres, Paris, PUF, 1980, p. 91.

3. M. Miaille, "Définir le droit", Droits, Revue française de théorie juridique, PUF, novembre 1990, p. 38 et suivantes .

4. A. Renaut, op. cit., p. 13. 
A fortiori, la question "qu'est-ce que le droit du travail?» ne saurait a priori revêtir un plus grand intérêt. En effet, tandis que le droit civil ou le droit pénal se présentent comme des œuvres de la raison, et forment une axiomatique qui déduit à partir de postulats de base un certain nombre de règles applicables à la société, le droit du travail s'est constitué par un "acte de remise en cause du modèle individualiste dont le Code civil représentait la systématisation juridique ${ }^{5}$. Le droit du travail n'est pas "une construction juridique monumentale édifiée mûrement par le législateur, mais plutôt une sédimentation de textes réalisés au hasard des flux et des reflux de la conjoncture politique et sociale ${ }^{6}$. Force est de reconnaître que le droit du travail "porte les traces des luttes dont il est issu"».

Mais une réflexion philosophique sur le droit du travail relève également de la gageure sur le plan déontologique. Le droit du travail est en effet l'objet de polémiques récurrentes et de tensions sociales extrêmement fortes. On se rapportera à titre d'exemple à un colloque organisé en 1986 sur le thème "Faut-il brûler le Code du travail?8", comme aux violentes réactions des partenaires sociaux à l'égard de chaque projet de loi en matière de législation sociale. Ainsi en prenant le droit du travail comme objet de réflexion, le philosophe se trouve face à une alternative dangereuse. Soit du haut de sa posture épistémologique, il décide de se placer en analyseur de concepts et de rester en dehors des faits qui nourrissent le droit du travail, et à l'écart des débats sociaux qui agitent son temps, mais il risque dès lors de se laisser enfermer dans une vision idéaliste du droit du travail, sans aucune prise directe sur le réel. Soit il décide d'établir un va-et-vient entre les faits et les concepts, en allant voir ce qui se passe concrètement sur le terrain du droit du travail et en prenant position dans des débats actuels, au risque de se perdre dans des évaluations éthiques et de devenir l'otage de querelles partisanes. Pour ces différentes raisons une interrogation philosophique sur le droit du travail est un défi à relever. C'est tout le sens de la déclaration, en guise de profession de foi, de Y. Schwartz. "S'il n'y a pas, dans l'invite à philosopher, de ségrégation entre les bons et les vils objets, il n'y a pas non plus de ségrégation entre l'exercice philosophique du passé et du présent :

5. Al. Supiot, "Délégalisation, normalisation et droit du travail», Droit social, 1984, $N^{\circ} 5$, p. 297.

6. Al. Supiot, "Le juge et le droit du travail», Droit social, $1980, N^{\circ} 5$, Numéro spécial, p. 60.

7. Ibid.

8. Colloque organisé à Montpellier, le 25 avril 1986. B. Teyssié, «Propos autour d'un projet d'autodafé", Droit social, 1986, № 7-8, p. 559-566. 
il serait contradictoire d'inventorier comment dans le passé les configurations contemporaines de la construction des grandes architectures conceptuelles ont pesé sur elles comme espaces de contraintes et d'opportunités à saisir, et de chasser dans l'exercice présent du métier la quête toujours obscure et difficile de ce qui nous rend disponible et nous provoque à penser, au nombre de quoi il $\mathrm{y} \mathrm{a}$ - ou il devrait y avoir - le travail - ou le non travail - des autres, et ce qui est en attente ou en souffrance d'y être pensé9».

\section{L'hypothèse de la révolution copernicienne}

L'observation des situations concrètes de travail donne le sentiment d'une transgression permanente du droit du travail. Alors que le travail reste un univers de contraintes juridiques ou extra-juridiques, l'expérience de la transgression semble inhérente à toute séquence industrieuse préalablement normée. Les récits d'Ivar Oddone (Redécouvrir l'expérience ouvrière) mettent en évidence des processus de mise en patrimoine de l'infraction efficace par lesquels les ouvriers enfreignent les normes et en inventent d'autres dans et par leur activité. Comment interpréter ces transgressions multiformes par lesquelles les sujets en permanence évaluent constamment l'univers normatif dans lequel ils évoluent c'est-à-dire confrontent les normes de leur milieu aux valeurs auxquelles ils donnent foi? Que nous apprennent ces transgressions du couple maudit du droit et des valeurs?

La réponse à ces questions nécessite d'accomplir une révolution copernicienne dans le domaine du droit du travail en posant comme hypothèse que le sujet de droit ne règle pas son activité sur le droit du travail, mais que, au contraire, il règle le droit du travail sur son activité en faisant plier les normes devant ses valeurs. Cette hypothèse a de quoi surprendre. Ne s'oppose-t-elle pas à la définition la plus élémentaire du droit? Le droit n'est-il pas ce qui doit lier le sujet d'une manière obligatoire? II nous faut tout d'abord préciser qu'un système juridique comprend non seulement des propositions normatives mais également des propositions mixtes dont la nature est à mi-chemin entre la norme et l'indication ${ }^{10}$. S. Romano signale dans le même sens que "les normes sont ou peuvent être une partie de

9. Le paradigme ergologique ou un métier de philosophe, Toulouse, Octarès, 2001, p. 49.

10. J.L. Gardies, "Système normatif et système de normes", in Archives de la philosophie du droit, tome XIX, 1974, p. 83. 
l'ordre juridique, mais restent bien loin de l'épuiser ${ }^{11}$ ». Toutes les propositions juridiques n'ont pas pour vocation d'imposer ou d'interdire un comportement, certaines d'entre elles sont purement indicatives. Dans ce cas il est compréhensible que l'acteur du travail choisisse dans l'offre juridique disponible les catégories qui lui permettront d'exercer au mieux son activité en fonction de critères de choix qui lui sont propres, en fonction des valeurs qu'il privilégie. Cette idée d'offre juridique dans laquelle les acteurs du travail, dont les gestionnaires, viendraient puiser, a été récemment mise en avant dans un appel à projets de recherche émanant de la DARES ${ }^{12}$.

Encore faut-il expliciter ce que nous entendons par l'affirmation selon laquelle le sujet règle le droit sur l'activité. Le verbe "régler" mérite d'être précisé car il peut prêter à confusion. II ne s'agit pas d'affirmer que le sujet crée le droit par son activité. Cela signifierait en effet que le fait et donc la force primerait sur le droit. Sauf à reconnaître que le droit n'est rien en dehors de la force et que seule la force crée le droit, l'idée que le sujet puisse créer des normes juridiques ex nihilo se heurte à de nombreux problèmes. Notre thèse ne serait au fond qu'une variante de la défense sophistique du droit du plus fort, illustrée par Thrasymaque ${ }^{13}$ ou de l'anarchisme stirnérien. L'auteur de l'Unique et sa propriété affirme en effet que "La force c'est le droit, le tigre qui m'attaque a raison et moi qui l'abats j'ai aussi raison". L'employeur qui exige de ses salariés qu'ils travaillent au-delà du maximum légal serait dans son droit à partir du moment où il disposerait de la possibilité économique d'imposer cette exigence à son personnel. Le droit serait en quelque sorte dissout dans le règne du subjectivisme et de l'arbitraire. Si le simple fait de transgresser une loi existante suffisait pour créer une nouvelle norme juridique, l'idée même de loi deviendrait inconcevable. Se confondant avec des impératifs éthiques, économiques ou physiques, le droit du travail perdrait sa raison d'être. II est possible de sentir à quel point cette idée est dangereuse.

Le verbe "régler» évoque plutôt le double mouvement d'interprétation et de validation auquel se livre nécessairement tout sujet de droit dans l'activité. II s'agit en effet de démontrer que la règle

11. S. Romano, L'Ordre juridique, p. 15.

12. La DARES est la Direction de l'Animation de la Recherche des Études et des Statistiques, elle est une composante qui dépend du Ministère de l'Emploi et de la Solidarité. Projet "Évolution des pratiques de gestion de l'emploi et droit du travail» 2001.

13. République, livre I, 336 b-347 e. L'affirmation initiale de Thrasymaque est que la justice consiste en l'intérêt du plus fort. 
juridique, nous dirons plus volontiers la loi entendue dans un sens large, n'acquiert sens, valeur et force contraignante qu'à partir du moment où le sujet a mené à son terme un processus double, plus ou moins conscient, de validation-interprétation, qui transforme la règle générale et abstraite en action singulière et concrète. En d'autres termes, le processus d'interprétation-validation transforme le droit mort (la lettre des textes juridiques) en droit vivant.

L'interprétation dépasse le cadre de l'application mécanique des règles. L'insuffisance chronique de la loi à subsumer l'ensemble des situations particulières sous une formule générale est un truisme de la philosophie politique depuis Platon ${ }^{14}$. Formulée d'une manière générale, la loi ne prend sens qu'à la suite d'une interprétation qui fait intervenir différents éléments, insérés dans une dynamique complexe, qu'il s'agira d'analyser en détail. L'idée générale qui préside à ce processus d'interprétation consiste à affirmer que le sujet détermine le sens d'une règle juridique en confrontant le texte de la loi à sa connaissance lacunaire du système juridique, à ses valeurs et aux impératifs de l'activité. L'activité est ainsi une dimension nécessaire à l'interprétation du droit. Ainsi l'article L 122-1 du Code du travaill5 dispose que "le contrat de travail à durée déterminée ne peut avoir ni pour objet ni pour effet de pourvoir durablement un emploi lié à l'activité normale et permanente de l'entreprise." Cet article fixe les conditions de recours au CDD, mais afin de pouvoir respecter cette disposition, l'employeur doit tout d'abord en décrypter le sens. II n'est pas évident de déterminer ce que le législateur entend par la notion "d'activité normale et permanente de l'entreprise». Certes, l'article $L$ 122-1-1 précise les trois seuls cas de recours au CDD strictement déterminés ${ }^{16}$. Mais l'abondante jurisprudence en la matière démontre à elle seule à quel point ce texte est sujet à des interprétations

14. Platon, Le Politique, $294 \mathrm{c}$.

15. Disposition relative au contrat à durée déterminée modifiée par la loi du 12 juillet 1990.

16. Le contrat de travail ne peut être conclu pour une durée déterminée que dans les cas suivants : $1^{\circ}$ ) Remplacement d'un salarié en cas d'absence, de suspension de son contrat de travail, de départ définitif précédant la suppression de son poste de travail ayant fait l'objet d'une saisine du comité d'entreprise ou, à défaut, des délégués du personnel, s'il en existe, ou en cas d'attente de l'entrée en service effective du salarié recruté par contrat à durée indéterminée appelé à le remplacer; $2^{\circ}$ ) accroissement temporaire de l'activité de l'entreprise; $3^{\circ}$ ) emplois à caractère saisonnier ou pour lesquels, dans certains secteurs d'activité définis par décrets ou par voie de convention ou d'accord collectif étendu, il est d'usage constant de ne pas recourir au contrat de travail à durée déterminée en raison de la nature de l'activité exercée et du caractère par nature temporaire de ces emplois. 
diverses et variées. Ainsi peut-on considérer qu'il soit possible de recruter par le biais de CDD successifs un salarié, dont la fonction permanente serait de remplacer le personnel absent de l'entreprise? La réponse à cette question est loin d'être évidente ${ }^{17}$. Le gérant de société ou le directeur des ressources humaines vont contribuer à construire le sens de cette règle à travers leurs pratiques de recrutement, pratiques qui découlent de leur vision de la loi construite dans et par l'activité et ses déterminants idéologiques et matériels ${ }^{18}$. Nous qualifierons l'ensemble de ces facteurs de «registre pluridimensionnel d'interprétation juridique».

Mais ce processus d'interprétation doit être associé à un processus de validation, qui consiste à déterminer non plus le sens mais la validité de la règle juridique. Là encore ce processus implique la combinaison de différents éléments ou critères que nous désignerons comme un «registre pluridimensionnel de validation métajuridique». Les philosophes ont en général examiné la question de la validation des règles juridiques sous deux aspects opposés. Soit ils considèrent que les critères de validation sont essentiellement formels et sont déterminables d'une manière dogmatique et a priori, soit ils considèrent au contraire que ces critères sont a posteriori et reposent sur les faits. II s'agit alors de déterminer si une règle est affectivement respectée ou non. Certains juristes et certains philosophes du droit, notamment anglo-saxons, s'emploient à démontrer depuis quelques années que ces deux approches sont inaptes à penser les modalités de validation des règles juridiques. Hart, Dworkin ou encore Perelman ont ainsi proposé différents modèles de raisonnement par lesquels les juges validaient ou invalidaient une règle juridique. Si leurs analyses sont fécondes en de nombreux points, l'hypothèse de la révolution copernicienne se démarque d'elles dans la mesure où d'une part la validation des règles juridiques fait intervenir des valeurs et des

17. Par exemple, un arrêt de la Cour d'appel de Limoges a estimé que quatre-vingtquatorze contrats de travail à durée déterminée conclus sur une période de quatre ans entre le même salarié et un même employeur ne pouvaient créer une relation de travail à durée indéterminée, dès lors qu'il sont tous été établis pour remplacer des salariés absents nommément désignés et pour l'exécution de tâches précises et temporaires, qu'aucun d'eux n'a été renouvelé sur plus de vingt-quatre mois et qu'ils n'ont pas eu pour objet de pourvoir durablement un emploi lié à l'activité normale et permanente de l'entreprise. CA limoges, 8/2/1993, Dalloz, 1993, Jurisprudence, note Jean Mouly, p. 431-434.

18. Quelle conception de la personne humaine est véhiculée par la culture d'entreprise? La législation est-elle stable ou instable en ce domaine? Quelle est l'évolution de la jurisprudence en ce domaine? Ce sont autant de facteurs, parmi tant d'autres, qui interviennent en ce qui concerne l'interprétation de la loi. 
critères parajuridiques qui relèvent de l'ordre éthique, économique et politique et où d'autre part, le processus de validation comme le processus d'interprétation ne sont pas le monopole des juristes, mais qu'ils sont attribuables à tous les acteurs du travail. Chaque sujet se livre par l'activité industrieuse à ce double processus d'interprétation et de validation des règles de droit. Alors que ce processus est mis en oeuvre consciemment par le juge et qu'il est plus ou moins formalisé, il peut relever de l'ordre du non-conscient et de l'informel pour les autres sujets de droit.

\section{L'exemple de la désobéissance à l'ordre illégal}

La désobéissance à l'ordre légal mais injuste (c'est-à-dire non conforme au droit naturel) nous permet d'exemplifier notre conception de la validation. La mémoire collective a élevé au rang de figure emblématique de l'histoire ceux qui avaient su désobéir et braver les lois qu'ils estimaient contraires au droit naturel. L'exemple des lois promulguées en Allemagne comme dans l'État de Vichy en vue de l'extermination du peuple juif est caractéristique. L'épuration ethnique était organisée par la loi et pourtant elle était en contradiction flagrante avec les droits de l'homme et du citoyen. Ce type de situation particulièrement dramatique dévoile la manière dont se nouent la loi et les valeurs dans le cadre du processus de validation. Le résistant refusait d'accorder une quelconque validité à une règle qu'il jugeait illégitime. Ce problème du conflit des devoirs envers l'État et envers la morale n'est pas criant de nouveauté. Sophocle en a fait la problématique de sa tragédie Antigone. En refusant de respecter le décret de Cléon, et en enterrant son frère, Antigone manifeste le peu de prix qu'elle accorde à une législation purement politique, qui s'oppose ouvertement aux impératifs moraux. Pour sa défense Antigone proclame la primauté des lois de la morale vis à vis de la législation humaine. "Oui, car ce n'est pas Zeus qui l'avait proclamée! Ce n'est pas la Justice, assise aux côtés des dieux infernaux; non ce n'est pas là les lois qu'ils ont jamais fixées aux hommes, et je ne pensais pas que tes défenses à toi fussent assez puissantes pour permettre à un mortel de passer outre à d'autres lois, non écrites, inébranlables des dieux! Elles ne datent, celles-là, ni d'aujourd'hui ni d'hier, elles sont éternelles, et nul ne sait le jour où elles ont paru. Ces lois-là, pouvaisje donc, par crainte d'une volonté humaine, m'exposer à leur vengeance devant les dieux? ${ }^{19}$ ". Antigone oppose ainsi à la loi

19. Sophocle, «Antigone», vers 450-460, Les Tragiques grecs, Tome 1, Paris, Robert Laffont, coll. Bouquins, 2001, p. 572. 
humaine contingente et arbitraire une autre loi d'une force supérieure, à savoir la loi religieuse.

Sans revêtir toujours ce degré dramatique, le problème de la validation des règles juridiques se pose quotidiennement dans le cadre des activités industrieuses et le double processus d'interprétation et de validation du droit est ce qui permet de transformer la loi écrite en un droit vivant et il constitue un facteur dynamique, véritable moteur, de l'évolution du droit du travail. Cette hypothèse nous conduit à élargir considérablement la notion d'ordre juridique, en retenant toute activité qui a un point visible de rattachement avec le monde des normes. Selon l'injonction programmatique de P. Orianne, «il faut prendre en considération non seulement les normes elles-mêmes et les rapports qu'elles entretiennent entre elles, mais aussi la totalité de l'espace où se déploie le droit, selon sa fonction, et observer notamment à ce titre les diverses activités auxquelles donnent lieu l'exécution, l'application et le contrôle des normes 20 ".

Le droit du travail, plus que tout autre domaine du droit, ne saurait être pensé à travers le schème réducteur normes - application des normes. Le champ des activités industrieuses génère des phénomènes de mises en dialogique des normes et des valeurs, qui débordent largement du cadre de l'interprétation standard de la philosophie du droit. Le droit du travail n'est pas uniquement un ensemble de normes juridiques que les acteurs du travail mettent en œuvre quotidiennement. II est un champ d'expérience et d'évaluation par les acteurs du travail de la manière dont la sphère des lois s'ordonne ou s'oppose aux valeurs d'égalité et de bien public, et aux sphères économique et politique. Si le droit du travail est toujours en arrière-fond dans chaque activité concrète de travail, il est sans cesse convoqué, interprété, et confronté avec les valeurs portées par l'individuel et le collectif. Une disposition du Code pénal prévoit que les commissaires aux comptes doivent révéler au Procureur de la République tout fait délictueux dont ils auraient connaissance lors de la réalisation de leurs opérations de contrôle. Les commissaires aux comptes engagent leur responsabilité pénale et civile s'ils ne respectent pas cette règle. Pourtant il apparaît que dans les faits peu de commissaires dénoncent leurs clients. En général, les commissaires préfèrent démissionner. En effet, le commissaire au compte, librement choisi par l'entreprise qu'il est chargé de contrôler, est également payé par son client. Révéler des faits compromettants sur

20. P. Orianne, Introduction au système juridique, Louvain-la-Neuve, Bruxelles, 1982, p. 130. 
l'entreprise dont il a la charge lui fera encourir le risque de perdre ce client, lors du prochain renouvellement de son mandat, mais il s'expose également à une mauvaise publicité, ce qui peut avoir des conséquences considérables sur le plan commercial. En outre, en dénonçant son client le commissaire aux comptes s'expose lui-même à des questions juridiques. Le procureur pourra ainsi s'interroger sur une éventuelle négligence ou complaisance passée du commissaire aux comptes. En général, les professionnels de la comptabilité préfèrent éviter toute relation avec la justice. Pourtant, se taire, c'est risquer une lourde peine, pouvant aller jusqu'à la prison; c'est en outre contraire aux règles déontologiques de la profession. La décision qui est prise convoque non seulement la connaissance que le sujet a du droit positif, mais également ses valeurs. Faut-il faire primer l'honnêteté et le respect de la justice, la fidélité vis à vis de son client ou au contraire les contraintes économiques qui pèsent sur l'exercice de son métier?

Chaque fois qu'une personne "contourne le règlement" (c'est-àdire enfreint la loi) en faisant primer ses valeurs ou les impératifs de l'activité sur la loi, elle ne nie pas le droit, elle ne le crée pas, elle lui donne son sens. Chaque situation de travail est porteuse de microdécisions qui se jouent parfois dans l'infinitésimal et qui donnent vie au droit du travail. Une règle générale et abstraite n'a que le sens conféré par que les acteurs du travail lui confèrent dans et par leur activité. Pour reprendre une expression de Y. Schwartz, le droit du travail se présente à la fois comme «un intermédiaire et un révélateur" des circulations de valeurs qui s'opèrent entre les organisations du travail et la sphère politico-juridique. Certes le droit du travail n'est pas le seul espace de capillarité entre les lois et les activités industrieuses, mais il concentre et il recueille à un haut degré l'expression et la mise à l'épreuve des valeurs politiques, éthiques et économiques de notre temps. Les corrections récurrentes dont le droit du travail est l'objet21, sensibles à travers la succession des lois et les revirements de jurisprudence, expriment à leur façon l'impossible anticipation des activités industrieuses par le droit. Aborder le droit du travail comme un ensemble de règles et de normes que les acteurs appliqueraient d'une manière plus ou moins scrupuleuse conduit irrémédiablement à une impasse méthodologique.

21. À titre d'exemple le régime des contrats à durée déterminée a été modifié cinq fois durant ces quinze dernières années. 
Au contraire, opérer un renversement de perspective, tel celui que nous proposons, devrait nous permettre de renouveler les termes de la question. La règle de droit, formulée par le législateur ou les partenaires sociaux, n'a que le sens conféré par les sujets de droit dans et par leurs activités. Dans ce jeu permanent entre le droit et les faits, le juge a un rôle fondamental à jouer car il est celui qui valide et qui oriente le sens que les sujets de droit donnent aux règles juridiques. Et ce sens est lié à un équilibre instable entre trois pôles : le pôle politique, le pôle économique, le pôle éthique. Mais le juge n'est pas le seul acteur à se livrer au processus d'interprétationvalidation. Nous pouvons imaginer ce que cette thèse a de dérangeant. Supposer que c'est le droit qui se centre sur l'activité via l'intervention des sujets nous conduit à affirmer que le droit ne devient proprement juridique qu'à partir du moment où il est mis en œuvre et confronté aux valeurs des sujets.

Christine Noël

Allocataire-Moniteur

Université de Provence 